\title{
Aplicación de un modelo hidromecánico de erosión interna asociado al esfuerzo en la fracción fina del suelo
}

\section{Application of a hydromechanical model for internal erosion associated with the stress in the soil finer fraction}

Fecha de entrega: 10 de enero 2015 Fecha de aceptación: 20 de mayo 2015

\section{Ricardo Moffat}

Departamento de Ingeniería Civil, Universidad de Chile, Blanco Encalada 2002, Santiago, Chile, rmoffatc@ing.uchile.cl

Se presenta un modelo teórico para determinar la frontera hidromecánica de un suelo internamente inestable sujeto a unflujo de agua vertical. El modelo se basa en ecuaciones de conservación de momentum y balance de masa, y considera que una muestra de suelo se divide en tres componentes: agua, fracción más fina y fracción más gruesa. Los parámetros del modelo son: esfuerzos efectivos, indice de poros, ángulo de fricción entre las fracciones gruesas y finas, y la proporción del esfuerzo efectivo que se transmite a la fracción más fina $G^{*}$. Comparamos la predicción de este modelo con resultados de ensayos de filtración en un permeámetro de grandes dimensiones, y demostramos que el modelo es capaz de representar adecuadamente lo observado en laboratorio. Adicionalmente se muestra que el valor de $G^{*}$ depende directamente del valor $D_{15}{ }^{\prime} / d_{85}$ 'del suelo estudiado. El modelo constituye una herramienta que se puede utilizar para deducir el valor del gradiente hidráulico crítico que gatilla la erosión interna en suelos no cohesivos susceptibles a este fenómeno.

Palabras clave: erosión interna, gradiente hidráulico crítico, medio granular, transmisión de fuerza
A theoretical model to determine the hydromechanical boundary of an internally unstable soil subject to vertical seepage. The model is based on momentum balance equations, which consider that the system is divided into three components: water, finer soil fraction, and coarser soil fraction. The parameters of the model are: the effective stress, the porosity of the soil, the friction angle between the coarse and fine fractions, and the proportion of the effective stress that is transmitted to the finer fraction $G^{*}$. Using laboratory data collected on a large permeameter, we demonstrate that the model is able to properly describe the observed behaviour. Furthermore, we show that the value of $G^{*}$ is related to the value of $D_{15}{ }^{\prime} / d_{85}$ ' and that it has the same trend as found experimentally. The proposed model is a promising method to deduce an approximate value of critical hydraulic gradient that triggers internal erosion in a cohesionless soil of known particle size distribution curve.

Keywords: internal erosion, critical hydraulic gradient, granular media, force transmission

\section{Introducción}

Fuerzas de filtración actúan en las partículas de suelo cada vez que un gradiente hidráulico genera flujo de agua. Estas fuerzas pueden afectar por erosión interna a suelos internamente inestables, ya que pueden producir el movimiento de la fracción más fina del suelo a través del esqueleto grueso del mismo suelo. En este artículo se considera que la erosión interna ocurre cuando la migración de partículas produce una reducción en el volumen total y el potencial consiguiente para el colapso de la matriz del suelo como se explica en Moffat et al. (2011). La inestabilidad interna se rige por: i) una restricción geométrica y ii) un umbral hidromecánico. Por lo tanto, un suelo que se considera potencialmente inestable como consecuencia de su curva granulométrica (restricción geométrica) exhibirá migración de partículas cuando las fuerzas inducidas por infiltración exceden un umbral crítico o límite hidromecánico, como se ha observado en experimentos de laboratorio (Skempton y Brogan, 1994; Moffat y Fannin, 2011; Chang y Zhang, 
2011) y simulaciones numéricas (Sari et al., 2011; Han y Cundall, 2013; Galindo-Torres et al., 2015).

El potencial de inestabilidad interna (restricción geométrica) se evalúa generalmente mediante métodos empíricos como el propuesto por Kezdi (1979) y Kenney y Lau $(1985,1986)$. En este trabajo se analiza de acuerdo al criterio de Kezdi, denominado como criterio de relación de filtro, en el cual requiere satisfacer la relación de $D_{15}$ \% $d_{85}{ }^{\prime} \leq 4$, que postuló Kezdi (1979) e independientemente De Mello (1975). Se trata de la división de la gradación en puntos arbitrarios a lo largo de la curva, para producir $D_{15}$ 'de la fracción gruesa y $d_{85}$ 'de la fracción fina. La premisa de este método es que existe un potencial para la inestabilidad siempre que $\left(D_{15}{ }^{\prime} / d_{85}{ }^{\prime}\right)_{\max }$ exceda 4 , de acuerdo con el criterio empírico para la retención del suelo de base por un filtro granular propuesto por Terzaghi (1939).

Es posible establecer el inicio de la inestabilidad como el gradiente hidráulico crítico $i_{c}$, cuando la migración de partículas comienza de acuerdo con los criterios explicados anteriormente. Por ejemplo, podemos utilizar los datos de ensayos de laboratorio para un suelo para estimar pares de valores de tensión media $\sigma$ ' y gradiente crítico local $i_{c}$ en el inicio de la inestabilidad interna. Estos valores generan una envolvente hidromecánica establecida experimentalmente por Moffat y Fannin (2011), que separa pares de valores ( $i$, $\left.\sigma^{\prime}\right)$ en estable e inestable. En ese trabajo se muestran los límites hidromecánicos promedio (además de los valores mínimo y máximo) obtenidos experimentalmente para 4 suelos diferentes (T-0, T-5, C-20 y C-30). Para valores de $\left(i, \sigma^{\prime}\right)$ por encima del límite hidromecánico se espera que el suelo presente erosión interna (inestable).

El objetivo principal de este estudio es proponer un modelo teórico simple para estimar el gradiente hidráulico crítico que gatilla el inicio de la inestabilidad interna en suelos no cohesivos. El modelo propuesto considera que solo una fracción del esfuerzo efectivo en la masa del suelo se transmite a la fracción más fina. De aquí en adelante, los granos con potencial para moverse a través de la estructura de poros de un suelo, se denominan fracción más fina del suelo que no corresponde necesariamente al material que pasa la malla \#200. La relación de tensiones entre fracción más fina y el esfuerzo efectivo aplicado a todo el suelo queda representado por el coeficiente $G^{*}$. Se ha observado que este valor es inversamente proporcional al valor de $D_{15}{ }^{\prime} / d_{85}$ ', según los datos de las pruebas de laboratorio publicados anteriormente (Moffat et al., 2011; Moffat y Fannin, 2011). El modelo propuesto debe ayudar a interpretar los datos de laboratorio y para estimar la posibilidad de erosión interna considerando los gradientes hidráulicos y esfuerzo efectivo del suelo en un proyecto de ingeniería.

\section{Formulación del modelo}

Se presenta un modelo basado en la teoría de medios porosos, para apoyar los conceptos sobre límites hidromecánicos de inestabilidad interna, y poder conciliar la aparente desconexión entre el ranking geométrico de inestabilidad interna y el ranking hidromecánico según lo descrito por Moffat y Fannin (2011). Muy pocos estudios han propuesto modelos físicos para determinar el inicio de la erosión interna en suelos (por ejemplo Indraratna y Radampola, 2002; Shire y O'Sullivan, 2013). Proponemos un nuevo modelo que considere el equilibrio de un sistema de tres componentes: agua, esqueleto del suelo, y la fracción más fina (teoría de mezclas). Las principales características de este modelo son: i) la capacidad de incluir análisis geométrico basado en la forma de la curva granulométrica, ii) la capacidad de incluir el valor de la tensión efectiva vertical y la variación de la tensión efectiva vertical en la zona de falla, iii) puede ser utilizado para modelar flujo ascendente o descendente, teniendo en cuenta el efecto de la fuerza de gravedad, iv) incluye los parámetros de resistencia del suelo como el ángulo de fricción, y v) la simplicidad. El modelo puede distinguir la tendencia general de los límites hidromecánicos para diferentes materiales y puede ser fácilmente incorporado a un código numérico. Este modelo sólo es aplicable cuando el suelo clasifica como potencialmente inestable según Kezdi (1979) o Kenney y Lau (1985) y cuando la fracción más fina está debajo del $35 \%$ en peso.

Como se muestra en la Figura 1, las principales hipótesis de este modelo son:

1) las fuerzas de inercia se desprecian, 2) las fuerzas de filtración actúan principalmente en las partículas de fracción más fina y 3) la tensión efectiva en la fracción más fina, es una fracción de la tensión efectiva total en la muestra. 


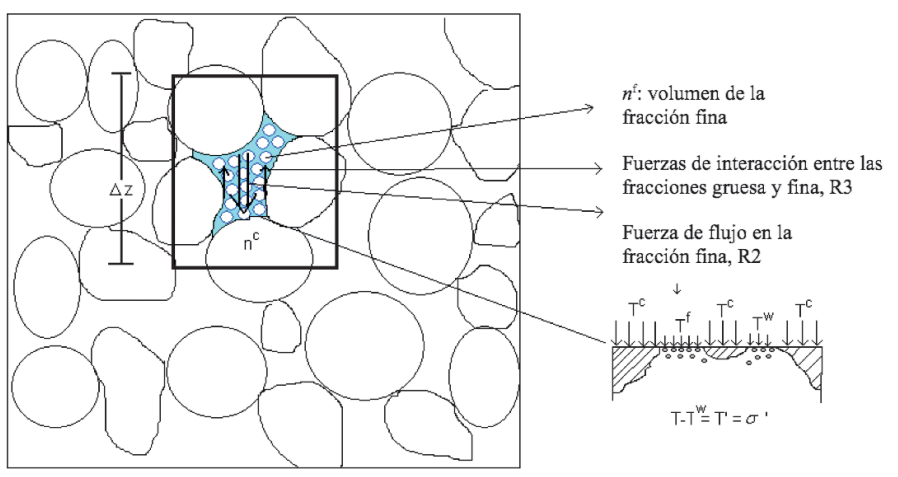

Figura 1: Volumen de control representativo usado para derivar el modelo

Aunque, no hay mucha evidencia experimental sobre la segunda suposición, se ha observado en simulaciones numéricas que los gradientes de presión son mucho más altos alrededor de las partículas de suelo más finas debido a la constricción del espacio de los poros y el consiguiente cambio en la permeabilidad (Sari et al., 2011; GalindoTorres et al., 2015). Galindo-Torres et al. modelaron partículas sólidas de dos tamaños diferentes observando que el gradiente hidráulico a lo largo del espacio ocupado por las partículas pequeñas es mayor y que la fuerza aumenta en estas partículas debido a la transferencia de momento desde el fluido a la fase sólida. Moffat et al. (2011) y Moffat y Fannin (2011) mostraron que variados parámetros influyen en la aparición de la inestabilidad interna. Por lo tanto, es extremadamente difícil la formulación de un modelo físico que se base en principios fundamentales y que refleje las mismas características observadas durante ensayos. Para la formulación de nuestro modelo, empezamos por considerar una unidad de volumen de suelo representativo que comprende tres componentes que pueden moverse de forma independiente, pero que influyen entre sí a través de fuerzas de interacción, como se muestra en la Figura 1 (Hassanizadeh y Gray, 1979). Entonces, la tensión total (en la dirección $z$ ) sobre el volumen de suelo es la suma de tres componentes:

$$
T=n T^{w}+n^{c} T^{c}+n^{f} T^{f}
$$

donde $T^{w}$ es la fracción de la tensión total que se transmite por el agua (presión de poros), $T^{c}$ y $T^{f}$ son la tensión parcial en las fracciones más gruesa y fina respectivamente. Los efectos volumétricos se consideran mediante el uso de valores de $n^{\mathrm{i}}$ que representan la fracción de volumen de cada componente. En consecuencia, las fracciones de volumen de estos componentes son: $n^{\mathrm{w}}=n$ : fracción de fluido (agua), o porosidad, $n^{\text {c }}$ : fracción del volumen de la parte gruesa $\left(=1-n-n^{f}\right), n^{f}$ : proporción del volumen de la fracción más fina, donde $n^{f}=\left(e / S_{f}\right) \cdot 100$ (Thevanayagam, 1998), donde $e$ es el índice de poros y $S_{f}$ es la proporción en peso de fracción más fina en \%.

El balance de momento puede ser utilizado para describir el límite de equilibrio de las tres fracciones. El balance de momento vertical de cada componente en la dirección vertical está dado por:

$$
\begin{aligned}
& \frac{\partial T^{w}}{\partial z}+\rho^{w} g=R 1-R 2+\rho^{w} \frac{\partial V_{w}}{\partial t} ; \text { agua } \\
& \frac{\partial T^{c}}{\partial z}+\rho^{c} g=R 3-R 1+\rho^{c} \frac{\partial V_{c}}{\partial t} ; \text { fracción gruesa } \\
& \frac{\partial T^{f}}{\partial z}+\rho^{f} g=-R 3+R 2+\rho^{f} \frac{\partial V_{f}}{\partial t} ; \text { fracción fina }
\end{aligned}
$$

donde $T^{i}$ es el esfuerzo en la componente " $i$ " $\left[\mathrm{ML} / \mathrm{T}^{2} / \mathrm{L}^{2}\right]$, $\rho^{i}$ es el peso unitario en la componente " $i$ " $\left(\rho^{c}=\rho^{f}=2.7\right.$ $\left.\mathrm{Mg} / \mathrm{m}^{3} ; \rho^{w}=1.0 \mathrm{Mg} / \mathrm{m}^{3}\right)$, g es la aceleración de gravedad $\left[\mathrm{L} / \mathrm{T}^{2}\right], V_{i}$ es la velocidad de la componente " $i$ " $[\mathrm{L} / \mathrm{T}], R 1$ es la fuerza de interacción entre el agua y fracción gruesa $\left[\mathrm{ML} / \mathrm{T}^{2} / \mathrm{L}^{3}\right], R 2$ es la fuerza de interacción entre el agua y fracción fina $\left[\mathrm{ML} / \mathrm{T}^{2} / \mathrm{L}^{3}\right]$ y $R 3$ es la fuerza de interacción entre la fracción fina y gruesa[ML/ $\left./ \mathrm{T}^{2} / \mathrm{L}^{3}\right]$.

En el caso de todos los ensayos analizados más adelante, se aplica lentamente al suelo el aumento de gradiente hidráulico promedio, por lo tanto, la tasa de cambio en la velocidad de filtración puede ser considerado muy bajo o insignificante $\left(\partial V_{\mathrm{w}} / \partial t=0\right)$. Además, se puede simplificar aún más el modelo suponiendo que las fracciones más gruesas y más finas no se mueven antes de la aparición de inestabilidad. Por lo tanto, las fuerzas de inercia se descuidan en este modelo y el último término en el lado derecho de las expresiones (2) a (4) es igual a cero.

Consideremos ahora las fuerzas de interacción $R 1, R 2$, y $R 3$ entre los tres componentes. $R 1$ y $R 2$ son función de la fuerza de la filtración por unidad de volumen, que puede ser aproximada por $i \gamma_{\mathrm{w}}$ (Terzaghi, 1939), donde $i$ es el gradiente hidráulico local en la capa de suelo que se está 
analizando. Además, se supone que debido a la diferencia en el tamaño de los granos finos y gruesos, la pérdida de carga se produce predominantemente en la fracción más fina del suelo, esto ha sido deducido anteriormente por Skempton y Brogan (1994). Por lo tanto, podemos hacer una simplificación adicional y asumir que las fuerzas de filtración actúan exclusivamente en la fracción más fina, y por lo tanto $R 1=0 \mathrm{y} R 2=i \gamma_{\mathrm{w}}$. Por otra parte, $R 3$ corresponde a la fuerza de interacción por unidad de volumen entre la fracción más fina y la fracción más gruesa de la tierra, se calcula directamente a partir de la teoría de Coulomb. Por lo tanto, después de cambiar los términos de (4) se obtiene la siguiente expresión para calcular $R 3$ :

$$
R 3=\frac{G^{*} \sigma_{v m}^{\prime} \tan \phi_{\mu}}{n^{f} \Delta z}
$$

donde $\Delta z$ es un largo unitario, $G^{*}$ es un factor de reducción de esfuerzos en la fracción fina (explicada más adelante) y $\phi_{\mu}$ es el ángulo de fricción verdadera entre la fracción fina y la gruesa.

Además, consideramos que la fracción fina transmite sólo una parte de la presión total efectiva $\sigma^{\prime}{ }_{v m}$ aplicada sobre la muestra internamente inestables. La misma hipótesis ha sido previamente planteada por Skempton y Brogan (1994), quienes postularon que 'en las mezclas arena-grava la mayor parte de la carga se transfiere a las partículas de grava, dejando la mayor parte de la arena bajo relativamente pequeñas presiones'. Ellos plantearon la hipótesis que la relación entre la tensión efectiva en la porción más fina y la tensión efectiva queda dada por un factor de reducción del esfuerzo $\alpha$, que depende de la curva granulométrica. Esta hipótesis también se ha verificado a través de simulaciones numéricas (por ejemplo Voivret et al., 2009). En lo que sigue se supone que:

$$
T^{f}=G^{*} \sigma_{v m}^{\prime}
$$

donde $G^{*}$ es un factor de base geométrica que determina la proporción de la tensión transferida a las partículas de la fracción fina (como lo hace el valor de $\alpha$ introducidas por Skempton y Brogan). La notación para la tensión vertical efectiva $\sigma_{v m}^{\prime}$ se ha utilizado para referirse a la labor previa de Skempton y Brogan y también de Moffat y Fannin (2011). Luego, de las ecuaciones (2) a (6) el gradiente hidráulico crítico puede ser estimado como: para flujo descendente

$$
i_{c r}=\frac{G^{*}}{\gamma_{w} \Delta z}\left(\sigma_{v}^{\prime} \tan \phi_{\mu}+\Delta \sigma_{v}^{\prime}\right)-\frac{n^{f} \rho_{f} g}{\gamma_{w}}
$$

o para flujo ascendente

$$
i_{c r}=\frac{G^{*}}{\gamma_{w} \Delta z}\left(\sigma_{v}^{\prime} \tan \phi_{\mu}+\Delta \sigma_{v}{ }^{\prime}\right)+\frac{n^{f} \rho_{f} g}{\gamma_{w}}
$$

\section{Aplicación del modelo propuesto a datos experimentales}

Moffat y Fannin (2011) analizaron los datos de las pruebas realizadas con un permeámetro y con cuatro gradaciones diferentes de suelo y valores de $D_{15}{ }^{\prime} / d_{85}$ '. La Tabla 1 resume las propiedades de estos suelos y la Tabla 2 contiene los valores de $i_{\text {cr }}$ y $\sigma$ ' en el inicio de la condición de falla observada en esos experimentos.

Tabla 1: Información para el análisis de estabilidad potencial de los suelos estudiados

\begin{tabular}{|c|c|c|c|}
\hline Suelo & $\begin{array}{c}\text { Contenido de } \\
\text { finos, } \%<74 \mu \mathrm{m}\end{array}$ & $\left(D_{15}{ }^{{ }^{\prime}} / d_{85}{ }^{\prime}\right)_{\max }$ & $\begin{array}{c}(\mathrm{H} / \mathrm{F})_{\min }{ }^{1} \\
\mathrm{~F}<0.2\end{array}$ \\
\hline T-0 & 0 & $13.7 @ 30 \%$ & 0.95 \\
\hline T-5 & 5 & $14.3 @ 30 \%$ & 0.90 \\
\hline C-20 & 20 & $7.7 @ 5 \%$ & 0.67 \\
\hline C-30 & 30 & $10 @ 5.6 \%$ & 0.67 \\
\hline
\end{tabular}

${ }^{1}$ Kenney y Lau (1986)

Además, el verdadero ángulo de fricción entre ambas fracciones se estima como $\phi_{\mu}=35^{\circ}$ para todos los materiales ensayados. Si bien es difícil obtener una evaluación fiable de $\phi_{\mu}$ (Mitchell, 1993), el valor supuesto de $35^{\circ}$ es razonable para minerales de cuarzo y feldespato. Sustituimos el gradiente hidráulico crítico $i_{\mathrm{cr}}$ junto con otros parámetros medidos en los experimentos realizados por Moffat y Fannin (2011) en (7) y (8) según corresponda el tipo de flujo (ver datos en Tabla 2). Este procedimiento produce un valor de $G^{*}$ que se asume único para cada suelo e independiente del nivel de tensión, de la misma manera como Skempton y Brogan (1994) suponen que el valor de $\alpha$ es único para cada suelo.

En la Figura 2 se muestra una comparación entre los valores calculados de $i_{\text {cr }}$ y $\sigma^{\prime}$ en el inicio de la falla y los valores medidos en el laboratorio. Con un valor constante de $G^{*}$ para cada suelo el modelo produce diferentes límites 
hidromecánicos para materiales del núcleo y de transición, lo cual es consistente con los resultados obtenidos experimentalmente.

Tabla 2: Datos experimentales usados para validar el modelo propuesto $\left(\phi_{\mu}=35^{\circ}\right)$

\begin{tabular}{|l|c|c|c|c|c|c|c|}
\hline \multicolumn{1}{|c|}{ Ensayo } & $G^{*}$ & $n^{\mathrm{f}}$ & $\begin{array}{c}\Delta \sigma_{\mathrm{vm}}{ }_{\mathrm{kPa}}, \\
\mathrm{kPa}\end{array}$ & $\Delta z, \mathrm{~m}$ & $\begin{array}{c}\sigma_{\mathrm{vm}}, \\
\mathrm{kPa}\end{array}$ & $\begin{array}{c}i_{\text {cr }} \\
\text { lab. }\end{array}$ & $\begin{array}{c}i_{\text {cr }} \\
\text { modelo }\end{array}$ \\
\hline T-0-25-D & 0.145 & 0.269 & 2.5 & 0.125 & 38.1 & 7.0 & 7.9 \\
\hline T-0-100-D & 0.145 & 0.279 & 12.6 & 0.125 & 62.1 & 12.2 & 9.1 \\
\hline T-0175-D & 0.145 & 0.279 & 31.6 & 0.125 & 182.6 & 25.4 & 30.6 \\
\hline T-5-25-D & 0.140 & 0.290 & Sin falla & & & & \\
\hline T-5-175-U & 0.140 & 0.290 & 64.5 & 0.125 & 101.1 & 58.4 & 49.2 \\
\hline T-5-50-D & 0.140 & 0.287 & -4.9 & 0.125 & 57.7 & 18.2 & 14.5 \\
\hline T-5-50-D(R) & 0.140 & 0.289 & -45.9 & 0.125 & 105.6 & 57.0 & 41.5 \\
\hline T-5-25-U & 0.140 & 0.284 & 4.1 & 0.05 & 22.87 & 13.3 & 18.1 \\
\hline T-5-30-U & 0.140 & 0.285 & 17.3 & 0.125 & 41.16 & 23.0 & 17.3 \\
\hline C-20-50-U & 0.200 & 0.153 & 24.4 & 0.125 & 24.6 & 35.2 & 41.5 \\
\hline C-20-85-U & 0.200 & 0.151 & 10.7 & 0.125 & 21.3 & 25.4 & 25.3 \\
\hline C-30-25-U & 0.170 & 0.180 & 11.7 & 0.125 & 15.9 & 14.7 & 16.7 \\
\hline C-30-50-U & 0.170 & 0.181 & 16.0 & 0.125 & 20.9 & 15.8 & 22.0 \\
\hline C-30-80-U & 0.170 & 0.191 & 37.2 & 0.125 & 41.4 & 39.2 & 44.9 \\
\hline C-30-100-U & 0.170 & 0.187 & 8.7 & 0.125 & 19.3 & 18.2 & 15.2 \\
\hline C-30-100-U & 0.170 & 0.187 & 34.6 & 0.125 & 40.9 & 39.3 & 43.6 \\
\hline
\end{tabular}

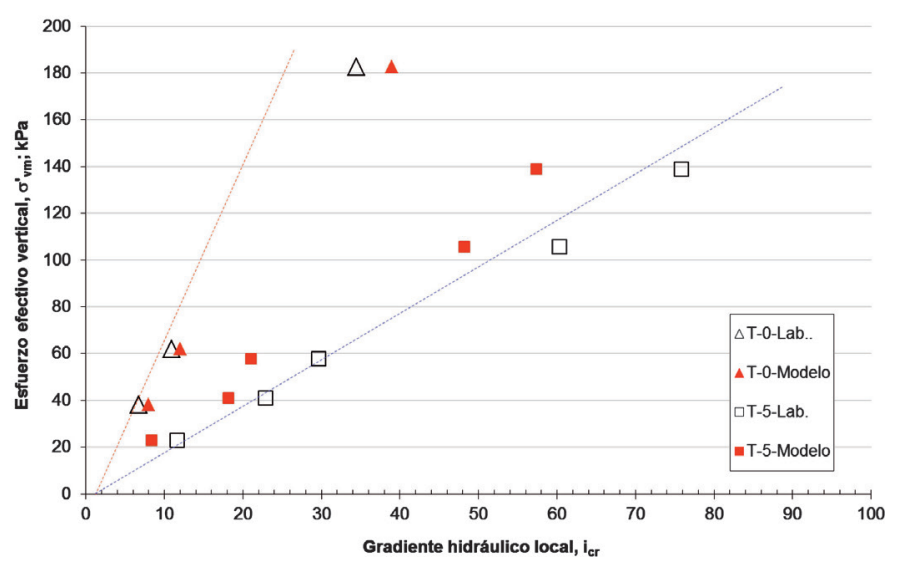

Figura 2: Comparación de los valores observados y estimados de $i_{\text {cr }}$

La Figura 3 muestra que los valores estimados basados en el factor geométrico $G^{*}$, se hacen más grandes cuando el correspondiente valor de $D_{15}{ }^{\prime} / d_{85}$ ' se hace más pequeño, lo que resulta en mayores valores de $i_{\text {cr }}$. La principal diferencia se produce al comparar los materiales de núcleo con materiales de transición. En contraste, la relación $D_{15}{ }^{\prime} /$ $d_{85}$ ' de los materiales T-0 y T-5, son muy similares (Moffat y Fannin, 2011) y, por lo tanto, el valor de $G^{*}$ no varía significativamente. Además, los valores de $n^{f}$ son muy similares para los dos materiales de transición (ver Tabla 2). En consecuencia, la diferencia en la pendiente de la frontera hidromecánica no se puede explicar solo a través de consideraciones geométricas y la variación en la tensión efectiva debe ser considerada $\Delta \sigma_{\mathrm{vm}}$ '. Además, los valores de $\alpha$ obtenidos por Skempton y Brogan (1994) también se representan en la Figura 3. Es posible ver que los valores de $\alpha$ obtenidos de forma independiente en suelos no cohesivos e inestables internamente siguen una tendencia similar de los valores de $G^{*}$ que se deducen de nuestro modelo. Interpretamos esta similitud como una validación del modelo propuesto.

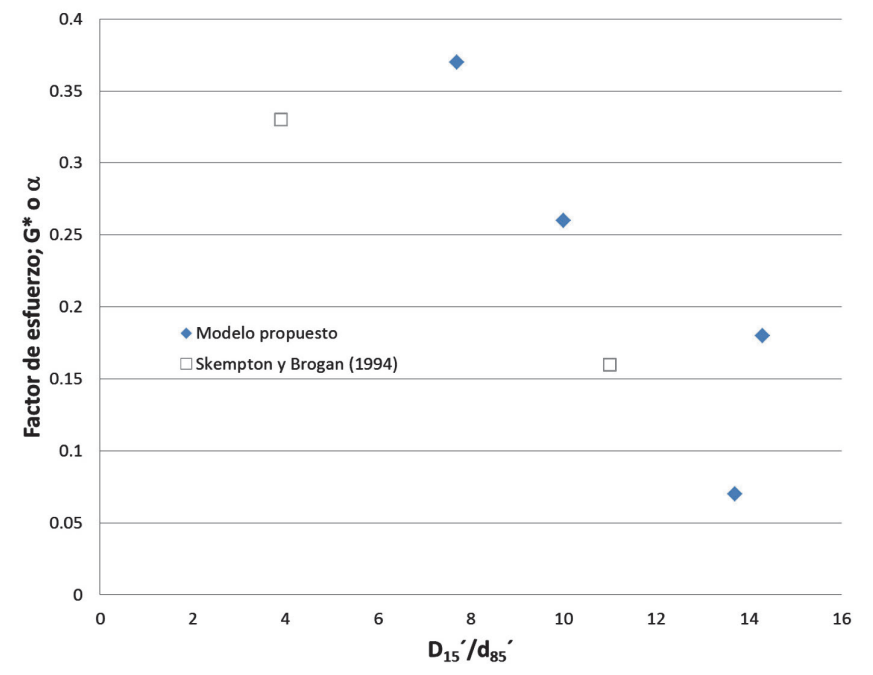

Figura 3: Factor de reducción de esfuerzo $G^{*}$ de acuerdo al modelo propuesto y según Skempton y Brogan (1984)

\section{Conclusiones}

Se deriva un modelo físico para deducir el gradiente hidráulico crítico en suelos no cohesivos y se aplica a datos experimentales previamente publicados (Moffat y Fannin, 2011). El modelo fue desarrollado teniendo en cuenta las fuerzas de interacción entre tres componentes: agua, fracción más fina, y fracción más gruesa; y tres supuestos: las fuerzas de inercia se desprecian, la tensión efectiva en la fracción más fina es una parte de la tensión efectiva en la muestra, y la fuerza de la filtración actúa sólo sobre las partículas de fracción más fina. Sobre la base de la aplicación del modelo teórico propuesto para analizar los datos experimentales de cuatro suelos no cohesivos, se concluye que:

(1) El esfuerzo efectivo y el gradiente hidráulico se consideran los parámetros más importantes para definir el 
inicio de la inestabilidad interna del suelo.

(2) El modelo identifica y explica la importancia de la variación de la tensión efectiva vertical $\Delta \sigma^{\prime}{ }_{v m}$ en la dirección de flujo, además de la influencia de la tensión media vertical efectiva $\sigma_{\mathrm{vm}}^{\prime}$ y el gradiente hidráulico $i_{\mathrm{jk}}$ a través de la zona de falla.

(3) El factor de reducción de la tensión en las partículas más finas $G^{*}$ disminuye a medida que el valor de $D_{15}$ '/ $d_{85}$ 'aumenta. Esto significa que para suelos que tienen valores de $D_{15}{ }^{\prime} / d_{85}{ }^{\prime}>4.0$ según Kezdi (1979), el gradiente hidráulico crítico $i_{\mathrm{cr}}$ disminuye a presión efectiva constante para valores más grandes de $D_{15}{ }^{\prime} / d_{85}{ }^{\prime}$.

(4) Los valores de $G^{*}$ son similares y siguen la misma tendencia que los valores del parámetro $\alpha$ previamente encontrado por Skempton y Brogan (1994) en ensayos de filtración ascendente sin carga externa aplicada al suelo.

Conocida la curva granulométrica de un suelo y la distribución de tensiones efectivas, es posible determinar el correspondiente valor de $G^{*}$ a partir de la Figura 3 para estimar el gradiente hidráulico crítico $i_{\text {cr }}$ usando las ecuaciones (7) y (8), que desencadena la inestabilidad interna. Por lo tanto, esperamos que el modelo propuesto sea un complemento útil de los actuales criterios para evaluar la estabilidad interna de los suelos en presencia de flujo de agua.

\section{Referencias}

Chang, D.S. and Zhang, L.M. (2011). A stress-controlled erosion apparatus for studying internal erosion in soils. Geotechnical Testing Journal 34(6), 579-589

De Mello, F.B. (1975). Some lessons learned from unsuspected, real and fictitious problems in earth dam engineering in Brazil. Proceedings 6th Regional Conference for Africa on Soil Mechanics and Foundation Engineering, Durban, South Africa, Sept. 1975, 285-304

Galindo-Torres, S.A., Scheuermann, A., Mühlhaus, H.B. and Williams, D.J. (2015). A micro-mechanical approach for the study of contact erosion. Acta Geotechnica 10(3), 357-368

Han, Y. and Cundall, P.A. (2013). LBM-DEM modeling of fluid-solid interaction in porous media. International Journal for Numerical and Analytical Methods in Geomechanics 37(10), 1391-1407
Hassanizadeh, M. and Gray, W.G. (1979). General conservation equations for multi-phase systems: 1. Averaging procedure. Advances in Water Resources 2: 131-144

Indraratna, B. and Radampola, S. (2002). Analysis of critical hydraulic gradient for particle movement in filtration. Journal of Geotechnical and Geoenvironmental Engineering 128 (4), 347-350

Kenney, T.C. and Lau, D. (1985). Internal stability of granular filters. Canadian Geotechnical Journal 22(2): 215-225

Kenney, T.C. and Lau, D. (1986). Reply: Internal stability of granular filters. Canadian Geotechnical Journal 23(3): 420-423

Kezdi, A. (1979). Soil physics. Amsterdam: Elsevier

Mitchell J. K. (1993). Fundamentals of Soil Behavior. $2^{\text {nd }}$ ed. John Wiley \& Sons

Moffat, R., Fannin, R.J, and Garner, S.J. (2011). Spatial and temporal progression of internal erosion in cohesionless soil. Canadian Geotechnical Journal 48(3), 399-412

Moffat, R. and Fannin, R.J. (2011). A hydromechanical relation governing internal stability of cohesionless soils. Canadian Geotechnical Journal 48(3): 413-424

Sari, H., Chareyre, B., Catalano, E., Philippe, P. and Vincens, E. (2011). Investigation of internal erosion processes using a coupled DEM-Fluid method. In Particles 2011 II International Conference on Particle-Based Methods, Oñate and Owen (eds.), Barcelona

Shire, T. and O'Sullivan, C. (2013). Micromechanical assessment of an internal stability criterion. Acta Geotechnica 8: 81-90

Skempton, A.W. and Brogan, J.M. (1994). Experiments on piping in sandy gravels. Géotechnique 44(3), 449-460

Terzaghi, K. (1939). Soil mechanics: a new chapter in engineering practice. $45^{\text {th }}$ James Forrest lecture. Journal Institution of Civil Engineers 12: 106-142

Thevanayagam, S. (1998). Effects of fines and confining stress on undrained shear strength of silty sands. Journal of Geotechnical and Geoenvironmental Engineering 123(6), 479-490

Voivret, C., Radjai, F., Delenne, J.-Y. and El Youssou, M.S. (2009). Multiscale force networks in highly polydisperse granular media. Physical Review Letters 102: 178001 